\title{
A NEW STRESS BASED TOPOLOGY OPTIMIZATION METHOD TO PREVENT STATIC AND DYNAMIC FAILURES OF DUCTILE OR BRITTLE MATERIAL
}

\author{
S. H. Jeong ${ }^{1}$, D. H. Choi ${ }^{2}$, G. H. Yoon ${ }^{3}$ \\ ${ }^{1}$ Department of Mechanical Engineering, Hanyang University \\ ${ }^{2}$ Department of Mechanical Engineering, Hanyang University \\ ${ }^{3}$ Department of Mechanical Engineering, Hanyang University (corresponding-author, \\ ghy@hanyang.ac.kr)
}

\begin{abstract}
A new stress-based topology optimization method (STOM) is developed in order to consider various static failure criteria such as the maximum shear stress (MSS) theory, the brittle Coulomb-Mohr (DCM) theory, and the modified Mohr (MM) theory. Because of nondifferentiability of failure criteria of these static failure theories, it seems that a successful topology optimization (TO) method considering the static failure has not been proposed yet. In order to solve TO problem which minimize the usage of material subject to the nondifferentiable static failure criteria, we formulate the differentiable failure criteria by using maximum and minimum operators. For a stable TO process, the p-norm stress measure approximating the maximum value of the stress norms and the adjustment parameter in the segregated design domain are implemented. Furthermore, a preliminary research considering the dynamic fatigue failure in the framework of the modified Goodman theory is presented. The validity and usefulness of the present STOM are demonstrated by solving typical TO benchmark problems.
\end{abstract}

Keywords: Stress-based topology optimization, static failure theories, ductile material, brittle material.

\section{INTRODUCTION}

This research paper presents a reliable stress-based topology optimization method (STOM) considering the static failure criteria for the brittle and ductile materials. Since the topology optimization (TO) for continuum stiff structures was introduced in the late 1980s, numerous works have investigated its theoretical and practical applications. Thus, several topology optimization methods, such as the homogenization based method [1, 2], the solid isotropic material with penalization (SIMP) method [3, 4], the level set method [5, 6], and the element connectivity parameterization (ECP) method [7, 8], have been developed and applied to a variety of engineering problems. However, few studies give enough investigation into the 
topology optimization of element-wise stress constraints as well as the various static and dynamic failure criteria, which are mathematically not differentiable with respect to both the TO design variables and the principal stresses. Consequently, this paper presents a new TO framework that utilizes the STOM for the static failures by introducing differentiable formulations of these static criteria using differentiable maximum and minimum operators.

\section{STRESS BASED TOPOLOGY OPTIMIZATION FOR NON DIFFERENTIABLE FAILURE CRITERIA}

\subsection{Topology optimization formulation with static failure criteria}

By following [9-11], the topology optimization to minimize material usage subject to failure criteria can be stated as follows.

$$
\begin{array}{cc}
\operatorname{Minimize}_{\gamma} & V(\tilde{\gamma})=\sum_{e=1}^{N E} \tilde{\gamma}_{e} v_{e} \quad(\tilde{\gamma}: \text { Filtered density }) \\
\text { subject to } & \left\langle g_{\max }\right\rangle_{1} \leq 1 \\
& \left\langle g_{\max }\right\rangle_{2} \leq 1 \\
\vdots & \\
\left\langle g_{\max }\right\rangle_{R N} \leq 1 \\
\tilde{\gamma}=\Xi(\gamma) \text { with the denstiy filter } \Xi \\
\left\langle g_{\max }\right\rangle_{k}=\max \left(g_{e}^{(O)}\right) \text { when } e \in \Omega_{k} \text { and the } e \text { th element exists. }
\end{array}
$$

where the maximum value of stress constraints in the $k$ th region is denoted by $\left\langle g_{\max }\right\rangle_{k}$ and the number of subdivided regions for the stress constraint evaluation is $R N$. In (2), the maximum operator is replaced by the $p$-norm approximation multiplied by a correction factor at the iterth optimization iteration as follows from [9-11]:

$$
\begin{gathered}
\left\langle g_{\max }\right\rangle_{k} \equiv c_{k}^{i t e r}\left\langle g_{P N}\right\rangle_{k} \quad\left(e \in \Omega_{k}\right) . \\
\left\langle g_{P N}\right\rangle_{k} \equiv\left(\sum_{e}\left(g_{e}^{(\odot)}\right)^{p} \tilde{\gamma}_{e}\right)^{1 / p} . \\
c_{k}^{i t e r}=\alpha \frac{g_{\max , k}^{i t e r-1}}{\left\langle g_{P N}\right\rangle_{k}^{i t e r-1}}+(1-\alpha) c_{k}^{i t e r-1} \quad 0<\alpha<1 .
\end{gathered}
$$

where $c_{k}^{\text {iter }}, g_{\max , k}^{\text {iter }}$ and $\alpha$ are the correction factor at the iterth optimization iteration, the real maximum value of the constraint functions in the $k$ th region, and the damping factor, respectively. The $p$ value is set to 4 in this research by following [9]. The damping factor, $\alpha$ used in equation (5), is fixed to a value of 0.5 by following the work in [9] . 


\subsection{Formulations of static failure criteria with phenomenological failure theories}

In this subsection, a short explanation and associated formulations of the static failure theories are presented. A thorough description of these failure theories can be found in [12]. In the case of ductile materials, there are three failure theories: the DE theory, the MSS theory, and the DCM theory. The criteria of these theories are given in (6-8). In the case of brittle materials, there are two failure theories: the MM theory and the BCM theory. The criteria of these theories are given in (9-13). Note that, the other criteria except for the criterion based on the DE theory are not differentiable with respect to principal stress due to maximum, minimum, and the logical if operator. Therefore the differentiable maximum and minimum operators among three principal stresses are developed [11].

The Distortion Energy (DE) Theory for Ductile Materials

$$
g_{e}^{D E}=\frac{\left[\sigma_{x}^{2}-\sigma_{x} \sigma_{y}+\sigma_{y}^{2}+3 \tau_{x y}^{2}\right]^{1 / 2}}{S_{y}} \leq 1 .
$$

The Maximum Shear Stress (MSS) Theory for Ductile Materials

$$
g_{e}^{M S S}=\frac{\max \left(\sigma_{1}, \sigma_{2}, \sigma_{3}\right)-\min \left(\sigma_{1}, \sigma_{2}, \sigma_{3}\right)}{S_{y}} \leq 1 .
$$

The Ductile Coulomb-Mohr (DCM) Theory for Ductile Materials

$$
g_{e}^{D C M}=\frac{\max \left(\sigma_{1}, \sigma_{2}, \sigma_{3}\right)}{S_{t}}-\frac{\min \left(\sigma_{1}, \sigma_{2}, \sigma_{3}\right)}{S_{c}}=\frac{\sigma_{1}}{S_{t}}-\frac{\sigma_{3}}{S_{c}} \leq 1 .
$$

The Brittle Coulomb-Mohr (BCM) Theory for Brittle Materials

$$
g_{e}^{B C M}=\frac{\max \left(\sigma_{1}, \sigma_{2}, \sigma_{3}\right)}{S_{u t}}-\frac{\min \left(\sigma_{1}, \sigma_{2}, \sigma_{3}\right)}{S_{u c}} \leq 1 .
$$

The Modified Mohr (MM) Theory for Brittle Materials

$$
\begin{gathered}
\sigma_{1} \leq S_{u t} \text { if } \sigma_{1} \geq \sigma_{2} \geq 0 \\
\sigma_{1} \leq S_{u t} \text { if } \sigma_{1} \geq 0 \geq \sigma_{2} \text { and }\left|\frac{\sigma_{2}}{\sigma_{1}}\right| \leq 1 \\
\frac{\left(S_{u c}-S_{u t}\right) \sigma_{1}}{S_{u c} S_{u t}}-\frac{\sigma_{2}}{S_{u c}} \leq 1 \text { if } \sigma_{1} \geq 0 \geq \sigma_{2} \text { and }\left|\frac{\sigma_{2}}{\sigma_{1}}\right|>1 \\
\sigma_{2} \leq-S_{u c} \text { if } 0 \geq \sigma_{1} \geq \sigma_{2}
\end{gathered}
$$


where, $S_{t}, S_{c}, S_{u t}$, and $S_{u c}$ represent the tensile yield strength, the compressive yield strength, the ultimate tensile strength, and the ultimate compressive strength, respectively.

\section{NUMERICAL EXAMPLES}

For the numerical examples, we consider an L-shaped beam and a cantilever beam problems. Note that, the method of moving asymptotes [15] is used as an optimizer.

\section{Example 1: L-bracket considering static failure theiroes}

For the first numerical example, we consider an L-shaped beam as shown in figure 1(a). This example has been widely used by many previous STOM research $[9,13,14]$ because it has a reentrant corner where stress concentration occurs. To test the MSS and DE theories, the material properties of the carbon steel 1018, a ductile metal, were used. Also, to test the BCM and MM theories, the material properties of the ASTM A48 gray iron 40, a brittle metal, were employed $[9,13,14]$. Because the BCM and the DCM theories share the same mathematical formulation, only the BCM theory was tested here. The detailed geometry, boundary condition, loading condition, and material properties are given in figure 1 . The number of subdomains $R N$ was fixed to eight for this example

Figure 2 shows the optimal topology layouts for each failure criterion. At the corner, the smooth boundary appears to avoid stress concentration in all layouts, as expected. Because the MSS theory is more conservative than the DE theory, the mass usage by the MSS failure criterion was larger than that by the DE failure criterion. Note that because the considered material from Figure 1(c) has a larger ultimate strength value in compression than that in tension, the thicker member is appeared at the upper right leg. Since the BCM theory is more conservative than the MM theory, the BCM model used more mass than the MM model.

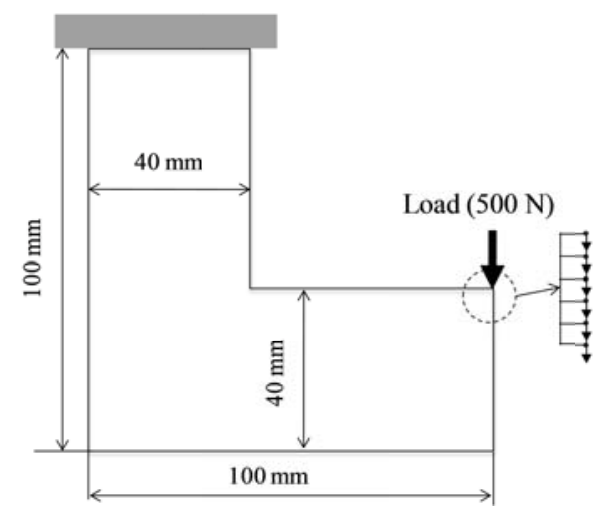

(a) 


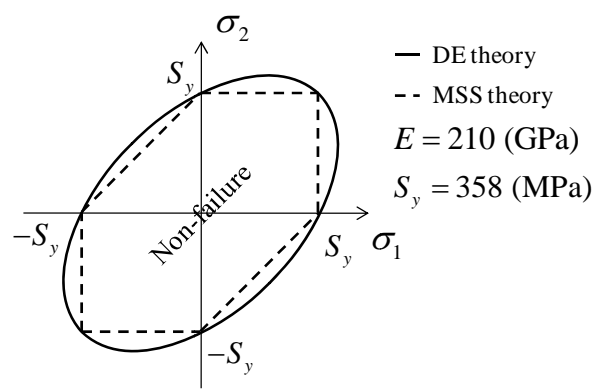

(b)

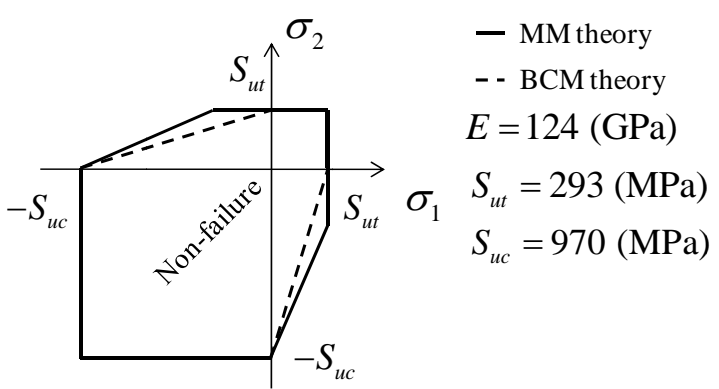

(c)

Figure 1. An L-shaped beam structure: (a) the geometry (The external load, $500 \mathrm{~N}$, was distributed on the six nodes of the tip of the right edge, $v=0.3$ ) (b) the material properties and the failure envelopes for the DE and MSS theories for carbon steel 1018, and (c) and the material properties and the failure envelopes for the BCM and MM theories for ASTM A48 gray iron 40.

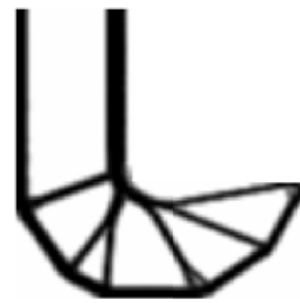

(a)

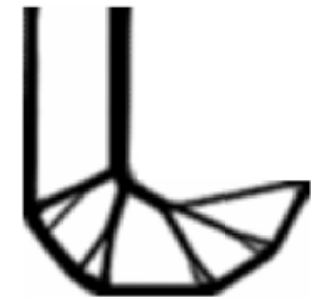

(b)

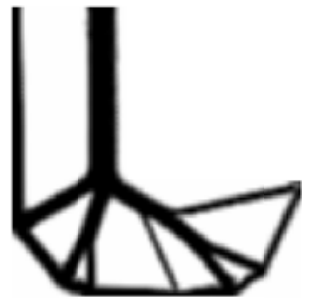

(c)

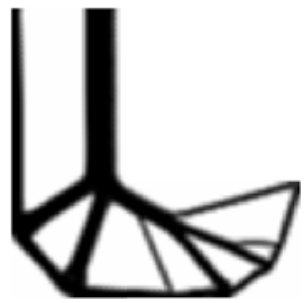

(d)

Figure 2. The optimized layouts of the L-shaped beam with the DE theory and the MSS theory ( $R N=8, n=3$ and $n_{\mathrm{s}}=0.5, \sigma_{\mathrm{A}}$ and $\sigma_{B}$ : unsorted principal stresses). (a) The layout using the DE theory $\left(V / V_{0}=0.293\right)$, (b) the layout using the MSS theory $\left(V / V_{0}=0.323\right)$, (c) the

layout using the MM theory ( $V / V_{0}=0.354$ ), and (d) the layout using the BCM theory

$$
\left(V / V_{0}=0.356\right) \text {. }
$$

\section{Example 2: Cantilever beam considering dynamic fatigue failure constraints (Modified}

\section{Goodman theory)}

Utilizing the theories developed above, we found that it is possible to consider the dynamic fatigue failure in the topology optimization problem minimizing the material usage subject to dynamic and static failure criteria; the detailed theory and optimization results will be reported in soon. To consider the dynamic fatigue effect, the static analysis and the dynamic analysis are considered in (14). In this research, $\left\langle L_{\max }\right\rangle_{k}$ in equation (14) is now the dynamic failure criterion based on the modified Goodman theory; the effects of the other dynamic criteria will be reported. Furthermore, $\left\langle g_{\max }\right\rangle_{k}$ in this problem is based on the DE theory (symmetric condition). To test our formulations, the numerical example of Figure 4 is considered. It is interesting that the anti-symmetric layout of figure 4 is obtained as the dynamic fatigue constraint $\left\langle L_{\max }\right\rangle_{k}$ ignores the compressive stress effect on the fatigue strength. It is also observed that the thick member is appeared in the upper part because the tensile stress is mainly applied to these areas. 


$$
\begin{array}{ll}
\underset{\gamma}{\operatorname{Minimize}} & V(\tilde{\gamma})=\sum_{e=1}^{N E} \tilde{\gamma}_{e} v_{e} \quad(\tilde{\gamma}: \text { Filtered density) } \\
\text { subject to } & \left\langle L_{\max }\right\rangle_{k}=\left(\sum_{e}\left(L_{e}\right)^{p} \tilde{\gamma}_{e}\right)^{1 / p} \leq 1, \quad\left(e \in \Omega_{k}, k=1, \cdots, R N\right) \\
& \left\langle g_{\max }\right\rangle_{k}=\left(\sum_{e}\left(\sigma / \sigma^{*}\right)^{p} \tilde{\gamma}_{e}\right)^{1 / p} \leq 1, \quad\left(e \in \Omega_{k}, k=1, \cdots, R N\right) \\
& \tilde{\gamma}=\Xi(\gamma) \text { with the denstiy filter } \Xi \\
& \mathbf{K}_{S} \mathbf{u}_{S}=\mathbf{F}_{S} \\
& \left(\mathbf{K}-\omega^{2} \mathbf{M}\right) \mathbf{u}_{D}=\mathbf{K}_{D} \mathbf{u}_{D}=\mathbf{F}_{D}
\end{array}
$$

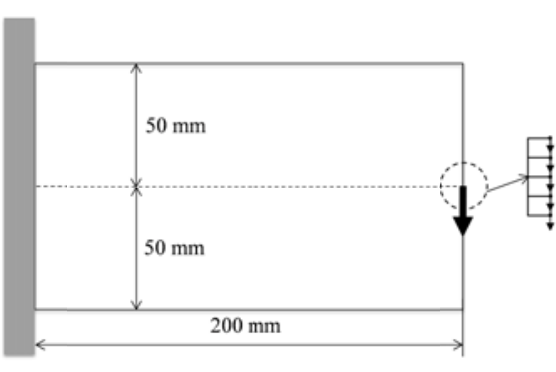

(a)

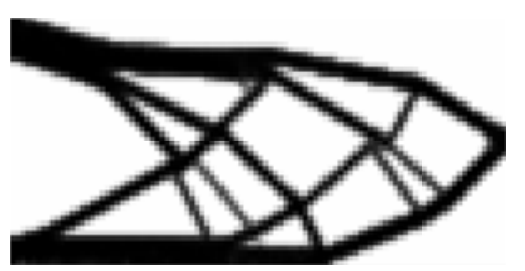

(b)

Figure 4. The geometry of a cantilever beam structure and the optimal layout considering the modifed Goodman theory. (The load was distributed on the five nodes of the tip of the right edge, $E=210 \mathrm{GPa}, v=0.3, \sigma_{u}=500 \mathrm{MPa}, \sigma^{*}=179 \mathrm{MPa}$, and $\rho=7820 \mathrm{~kg} / \mathrm{m}^{3}$, $\omega=0.0628 \mathrm{rad} / \mathrm{s}$, Static load: $F_{S}=300 \mathrm{~N}$ and Fully reversed dynamic load: $F_{D}=500 \mathrm{~N}$ ).

\section{CONCLUSIONS}

This research presents a new topology optimization framework that considering the various failure criteria, including the DE theory, the MSS theory, the DCM theory, the BCM theory, and the MM theory, by introducing differentiable maximum and minimum operators as well as a differentiable logical operator. These operators were essential for deriving the sensitivity analysis of these failure criteria with respect to both the topology optimization design variables and the principal stress values. Also, the preliminary research considering dynamic fatigue failure are represented. By solving several numerical examples, the validity and usefulness of the present method are verified.

\section{Acknowledgements}

This work was supported by the National Research Foundation of Korea (NRF) grant funded by the Korea government (MEST) (No. 2011-0016701).

Also, this work was supported by grants from "Development of the Prototyping Ball Bearings for a Rocket Turbopump” project of Ministry of Education Science and Technology (MEST). The authors thank to MEST. 


\section{REFERENCES}

[1] Suzuki K., Kikuchi N., “A homogenization method for shape and topology optimization”. Comput. Meth. Appl. Mech. Eng., 93, 291-318, 1991.

[2] Nishiwaki S., Frecker M. I., Min S. J., Kikuchi N., "Topology optimization of compliant mechanisms using the homogenization method”. Int. J. Numer. Methods Eng., 42, 535559, 1998.

[3] Bendsoe M. P., Sigmund O., “Material interpolation schemes in topology optimization”. Arch. Appl. Mech., 69, 635-654, 1999.

[4] Bendsoe M. P., Sigmund O., “Topology optimization: theory, methods, and applications”. Berlin; New York: Springer, 2003.

[5] Wang M. Y., Wang X. M., Guo D. M., “A level set method for structural topology optimization”. Comput. Meth. Appl. Mech. Eng., 192, 227-246, 2003.

[6] Mei Y. L., Wang X. M., “A level set method for structural topology optimization and its applications”. Adv. Eng. Softw., 35, 415-441, 2004.

[7] Yoon G. H., Kim Y. Y., "Element connectivity parameterization for topology optimization of geometrically nonlinear structures”. Int. J. Solids Struct., 42, 1983-2009, 2005.

[8] Yoon G. H., Kim Y. Y., “Topology optimization of material-nonlinear continuum structures by the element connectivity parameterization”. Int. J. Numer. Methods Eng., 69, 2196-2218, 2007.

[9] Le C., Norato J., Bruns T. Ha C., Tortorelli D., "Stress-based topology optimization for continua”. Struct. Multidiscip, Optim., 41, 605-620, 2010.

[10] Jeong S. H., Choi D. H., Yoon G. H., "Separable stress interpolation scheme for stressbased topology optimization with multiple materials". In preparation.

[11] Jeong S. H., Park S. H., Choi D. H., Yoon G. H., “Topology optimization considering static failure theories for ductile and brittle materials". In review.

[12] Budynas R. G., Nisbett J. K., "Shigley’s mechanical engineering design”. New York: Mcgraw-Hill, 2011.

[13] Paris J., Navarrina F., Colominas I., Casteleiro M., “Topology optimization of continuum structures with local and global stress constraints". Struct. Multidiscip, Optim., 39, 419-437, 2009.

[14] Paris J., Navarrina F., Colominas I., Casteleiro M., "Block aggregation of stress constraints in topology optimization of structures”. Adv. Eng. Softw., 41, 433-441, 2010.

[15] Svanberg K., "The method of moving asymptotes - a new method for structural optimization”. Int. J. Numer. Methods Eng., 24, 359-373, 1987. 